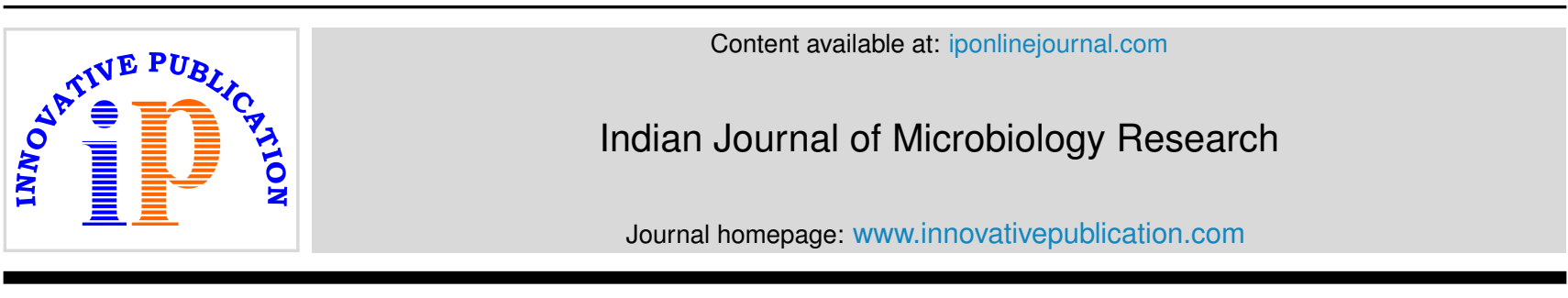

Original Research Article

\title{
Antimicrobial resistance surveillance of various clinical isolates in a diagnostic laboratory- An observational study
}

\author{
Chakrapani Kammineni' ${ }^{1}$, Naresh Yajamanam², Amarnath K Reddy ${ }^{2}$, A. Mahammed \\ Shafi ${ }^{1}$, Sreekanth Reddy Basireddy ${ }^{3, *}$ \\ ${ }^{1}$ Dept. of Microbiology, Bharathi Diagnostics, Kurnool, Andhra Pradesh, India \\ ${ }^{2}$ Dept. of Biochemistry, Bharathi Diagnostics, Kurnool, and Santhiram Medical College, Nandyala., Andhra Pradesh, India \\ ${ }^{3}$ Dept. of Microbiology, Kurnool Medical College, Kurnool, Andhra Pradesh, India
}

\section{A R T I C L E I N F O}

\section{Article history:}

Received 26-07-2019

Accepted 21-08-2019

Available online 09-09-2019

\section{Keywords:}

Gram positive organisms

Gram negative organisms

Local pattern Surveillance

\begin{abstract}
A B S T R A C T
Introduction: Infectious diseases are among the top most killers with great mortality and morbidity. Antibiotic prescription is a very common entity in a clinical setup. Because of the inadvertent and irrational use of antibiotics, increasingly drug resistance is being observed among the clinical isolates, which takes a great toll on the health, economical and social status of the affected. Multidrug resistance is observed among all the major bacterial species. Antimicrobial resistance surveillance should be done at local, regional, and national level in order to formulate the antibiotic policies which helps in guiding the clinicians in rational use of antibiotics. The present study emphasizes on systematic analysis of the Antimicrobial resistance surveillance of various clinical isolates in and around Kurnool which helps the local clinicians in the better management of the patients.

Aims and Objectives: The present study aims in identifying the etiological agents from various clinical samples and detecting their resistance pattern.

Materials and Methods: All the samples sent to the Microbiology department in Bharathi Diagnostics, Kurnool, Andhra Pradesh, during the study period of January 2018 to December 2018 were processed according to the standard protocols. Antimicrobial susceptibility testing was done for all the significant isolates by using Kirby Bauer disc diffusion method and the interpretation was carried out as per the CLSI guidelines. The most common clinical samples obtained were Urine, Blood, Pus/ Wound swab, Sputum and other Body fluids.
\end{abstract}

(C) 2019 Published by Innovative Publication.

\section{Introduction}

Infectious diseases are among the top most killers with great mortality and morbidity. Antibiotic prescription is a very common entity in a clinical setup. Because of the inadvertent and irrational use of antibiotics, increasingly drug resistance is being observed among the clinical isolates, which takes a great toll on the health, economical and social status of the affected. Drug resistance is observed among all the major bacterial species with Multidrug resistance commonly observed among nosocomial infections. ${ }^{1,2}$

\footnotetext{
* Corresponding author.

E-mail address: basikanthu@gmail.com (S. R. Basireddy).
}

India is one of the largest consumer of antibiotics in the world and also carries highest burden of drug resistance pathogens. Irrational and over use of antibiotics have been the culprits for drug resistance in India. With the emergence and rapid spread of metallobetalactamase producers like NDM-1 in India, only very few alternative antibiotics are available for the treatment. As there is scarcity of the national data, Indian council of medical research initiated Antimicrobial Resistance Surveillance \& Research Network (AMRSN) in the year 2013 which covers antibiotic resistance surveillance of major bacterial groups. Increasing resistance of gram negative isolates has been observed by this network. Alarming rates of resistance to fluoroquinolones, third generation cephalosporins was 
observed. Increased carbapenem resistance is particularly worrisome. $^{2}$

Antimicrobial resistance surveillance should be done at local, regional, and national level in order to formulate the antibiotic policies which helps in guiding the clinicians in rational use of antibiotics which decreases the mortality and morbidity and in turn the emergence of drug resistance. Knowledge about the local drug resistance pattern is extremely important for the clinician in order to choose the appropriate empirical antibiotic. The present study emphasizes on systematic analysis of the Antimicrobial resistance surveillance of various clinical isolates in and around Kurnool which helps the local clinicians in the better management of the patients.

\section{Aims and Objectives}

The present study aims in identifying the etiological agents from various clinical samples and detecting their resistance pattern.

\section{Materials and Methods}

All the samples sent to the Microbiology department in Bharathi Diagnostics, Kurnool, Andhra Pradesh, during the study period of January 2018 to December 2018, were processed according to the standard protocols. Antimicrobial susceptibility testing was done for all the significant isolates by using Kirby Bauer disc diffusion method and the interpretation was carried out as per the CLSI guidelines. ${ }^{3}$ The most common clinical samples obtained were Urine, Blood, Pus/ Wound swab, Sputum and other Body fluids.

\section{Results}

A total of 5687 samples were processed during study period out of which 1696 samples were culture positive. Most common sample was Urine, followed by Pus/ Wound swab, Blood, Sputum and Body fluids.

\subsection{Urinary tract infections}

Out of 3,164 urine samples obtained 941 have shown significant growth. The distribution of the various bacterial pathogens among the urine clinical isolates were as follows

\subsection{Wound infections}

Out of 573 pus/ wound swab samples obtained 235 were culture positive. The distribution of the various bacterial pathogens among the clinical isolates were as follows

\subsection{Blood cultures}

A total of 821 blood cultures were obtained during the study period out of which 166 samples have shown positive blood

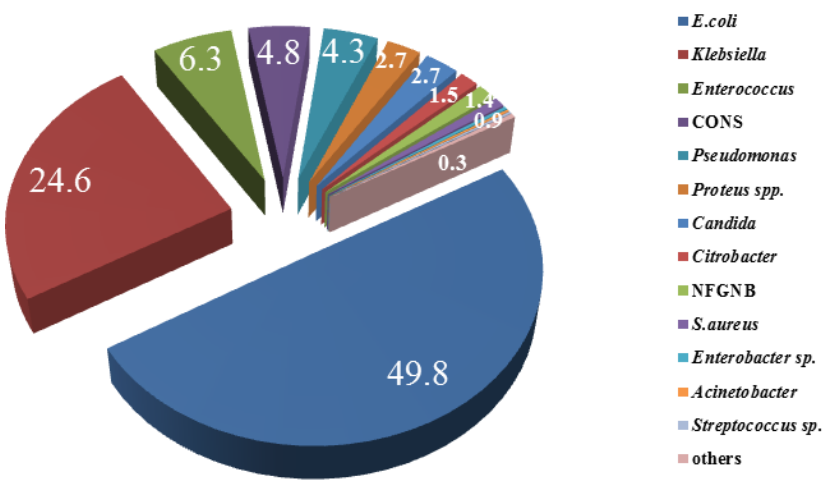

Fig. 1: Distribution of the various bacterial pathogens among the urine clinical isolates

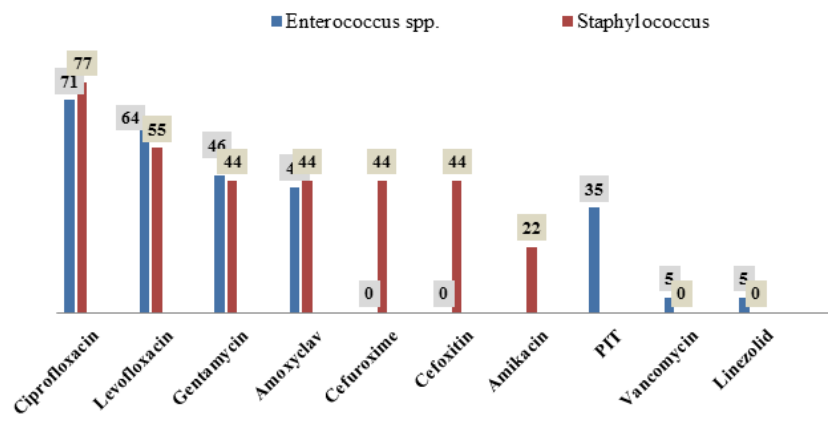

Fig. 2: Resistant pattern of Enterococcus spp. and Staphylococcus spp. isolated from the urinary tract infections

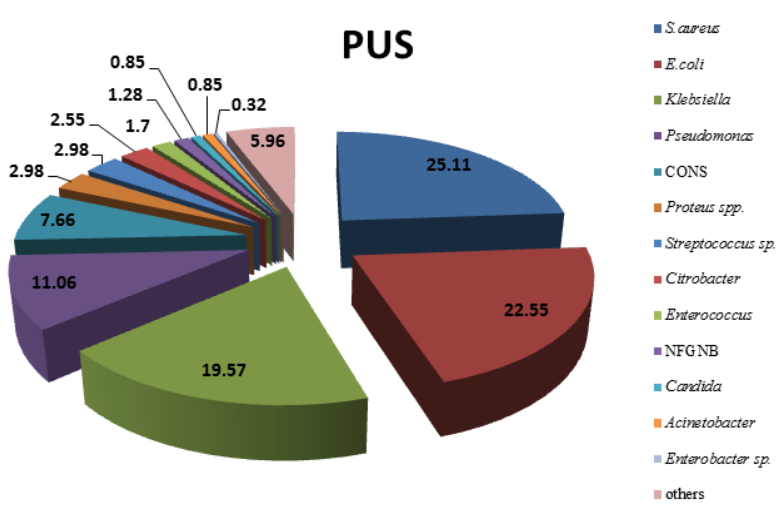

Fig. 3: Distribution of the various bacterial pathogens among the pus specimens 
Table 1: Resistant pattern of common urinary pathogens isolated during the study period

\begin{tabular}{|c|c|c|c|c|}
\hline Urine & E.coli & Klebsiella spp. & Proteus spp. & Pseudomonas \\
\hline Amoxyclav & 81.86 & 89.64 & 52.94 & - \\
\hline Ceftriaxone & 69.87 & 67.12 & 29.41 & - \\
\hline Ciprofloxacin & 67.61 & 63.06 & 23.53 & 25 \\
\hline Co-trimoxazole & 60.39 & 63.2 & 76.47 & - \\
\hline CFS & 32.89 & 42.73 & 5.88 & 40 \\
\hline Gentamycin & 29.34 & 37.98 & 29.41 & 22.5 \\
\hline Fosfomycin & 23.02 & 34.24 & 29.41 & - \\
\hline Nitrofurantoin & 14.76 & 36.65 & - & - \\
\hline PIT & 19.69 & 23.32 & 5.88 & 25 \\
\hline Amikacin & 12.45 & 18.5 & 5.88 & 22.5 \\
\hline Imipenem & 4.12 & 7.23 & 0 & 2.5 \\
\hline Meropenem & 3.39 & 5.85 & 0 & 2.5 \\
\hline Tigecycline & 0 & 0 & - & - \\
\hline Colistin & 0 & 0 & 100 & 0 \\
\hline
\end{tabular}

Table 2: Resistant pattern of common pathogens isolated from the wound infections during the study period

\begin{tabular}{llllll}
\hline PUS & E.coli & Klebsiella spp. & Pseudomonas & Proteus spp. & Others \\
Amoxyclav & 86.79 & 91.3 & NA & 77.78 & 81.82 \\
Ceftriaxone & 71.7 & 71.74 & NA & 66.67 & 81.82 \\
Ciprofloxacin & 73.58 & 60.87 & 23.08 & 44.44 & 36.36 \\
Co-trimoxazole & 73.58 & 67.39 & NA & 66.67 & 54.55 \\
CFS & 30.19 & 36.96 & 42.31 & 11.11 & 9.09 \\
Gentamycin & 35.85 & 41.3 & 26.92 & 55.56 & 54.55 \\
Fosfomycin & 44 & 48 & NA & - & - \\
Aztreonam & 73.58 & 60.87 & 34.62 & 55.56 & 81.82 \\
PIT & 30.19 & 39.13 & 34.62 & 11.11 & 18.18 \\
Amikacin & 16.98 & 23.91 & 19.23 & 22.22 & 36.36 \\
Imipenem & 1.89 & 10.87 & 7.69 & 0 & 0 \\
Meropenem & 1.89 & 10.87 & 3.85 & 0 & 0 \\
Tigecycline & 0 & 0 & - & 100 & 0 \\
Colistin & 0 & 0 & 0 & 100 & 0 \\
\hline
\end{tabular}

Table 3: Distribution of the various bacterial pathogens among the blood clinical isolates

\begin{tabular}{lll}
\hline Type of pathogen From Blood Cultures $(\mathbf{N}=\mathbf{1 6 6})$ & No. of isolates & \% isolated \\
E.coli & 31 & 18.67 \\
CONS & 30 & 18.07 \\
Klebsiella & 28 & 16.81 \\
Pseudomonas & 22 & 13.25 \\
S.aureus & 16 & 9.63 \\
NFGNB & 09 & 5.42 \\
Acinetobacter & 08 & 4.81 \\
Candida & 06 & 3.41 \\
Enterococcus & 05 & 3.01 \\
Salmonella Typhi & 02 & 1.20 \\
Proteus spp. & 02 & 1.20 \\
Others & 07 & 4.21 \\
\hline
\end{tabular}


Table 4: Resistant pattern of common blood pathogens isolated during the study period

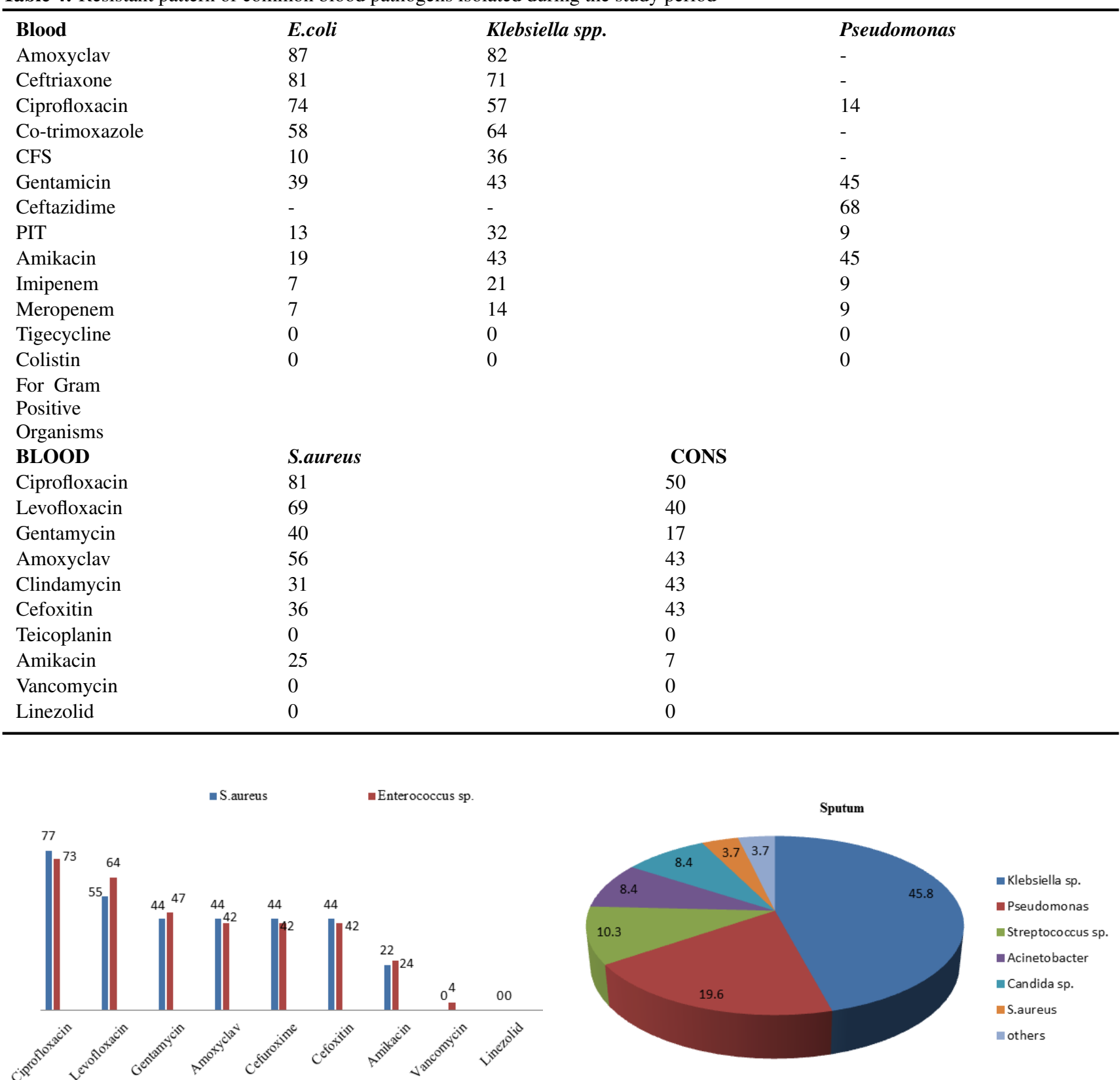

Fig. 4: Resistant pattern of S.aureus and Enterococcus spp. isolated from the wound infections

culture.

\subsection{Sputum}

\section{Discussion}

A phenomenal increase in the drug resistance has been observed recently among the clinical isolates which ranged from resistance to a few antibiotics to almost all the available antibiotics (MDR, XDR, PDR ). In the
Fig. 5: Distribution of the various bacterial pathogens among the Sputum specimens

present scenario of evidence based medicine the role of microbiology services have gained much importance compared to the earlier days. Better understanding of the microbial aspects along with sound knowledge of pharmacokinetics and pharmacodynamics of the antibiotics helps the clinicians in choosing the wide variety of antibiotics appropriately for the better management of patients. Local antibiotic resistance patterns play a crucial role in this. ${ }^{4,5}$ 
Table 5: Resistant pattern of common pathogens isolated from sputum specimens during the study period

\begin{tabular}{llll}
\hline Sputum & Klebsiella spp. & Pseudomonas & Acinetobacter sp. \\
Amoxyclav & 87.5 & NA & 100 \\
Ceftriaxone & 62.5 & NA & 100 \\
Co-trimoxazole & 64.3 & NA & 88.9 \\
Ciprofloxacin & 48.2 & 23.8 & 100 \\
Gentamycin & 44.6 & 19.1 & 88.9 \\
CFS & 41.1 & 9.5 & 77.8 \\
PIT & 41.1 & 19.1 & 88.9 \\
Amikacin & 32.1 & 19.1 & 88.9 \\
Imipenem & 12.5 & 3.2 & 55.7 \\
Meropenem & 8.7 & 3.2 & 55.7 \\
Tigecycline & 0 & - & 0 \\
Colistin & 0 & 0 & 0 \\
\hline
\end{tabular}

In the present study, majority of the isolates were obtained from urine samples followed pus/ wound swab and sputum. It is a known fact that E.coli is the most common causative agent of UTI, the present study E.coli constituted half of the total urinary isolates followed by Klebsiella sp. \& Enterococcus sp. E.coli was highly resistant to Amoxyclav followed by $3^{\text {rd }}$ gen Cephalosporins (cefixime, ceftri axone, cefotaxime), Fluoroquinolones (Ciprofloxacin, Norfloxacin, Levofloxacin) and Co-trimoxazole making majority of the orally available antibiotics ineffective for treatment. The best oral antibiotics with least resistance in the present study were Fosfomycin $(23.02 \%)$ \& Nitrofurantoin(14.76\%). In a study conducted by Sardar et al similar findings were observed where all the isolates were susceptible to fosfomycin on par with the carbapenems where as nitrofurantoin resistance was also considerably low $(17.7 \%)^{6}$ The other antibiotics like piperacillin/tazobactum, cefaperazone/sulbactum and Amikacin have shown significant activity against the E.coli isolates. The high end antibiotics like colistin, Carbapenems (IPM, MRP) have shown the greatest activity with almost all the isolates being susceptible to them. Though Tigecycline had also shown absolute sensitivity in vitro, it is not a drug of choice for UTIs as the urinary levels are low. Klebsiella has also shown almost similar resistance pattern as that of E.coli. In contrast to Enterobacteriaceae members Pseudomonas is not susceptible to routine antibiotics like Amoxyclav, ceftriaxone, Cotrimoxazole, Tetracyclines, Nitrofurantoin, etc. Among Pencillins, only ureido-pencillins (Piperacillin, Ticarcillin), among Cephalosporins only ceftazidime, Cefaperazone \& Cefepime have got the anti- pseudomonal activity which can be used for the treatment. Polymyxins, Carbapenems \& Aminoglycosides are having highest anti- pseudomonal activity. Pseudomonas was absolutely sensitive to colistin \& polymyxin B followed by Aminoglycosides. Among the Fluoroquinolones, Ciprofloxacin has shown the greatest activity against Pseudomonas with only $1 / 4^{\text {th }}$ of the isolates being resistant.
Among the Gram positive organisms, methicillin resistance was observed in $44 \%$ of the Staphyloccocal isolates. Levofloxacin has shown better activity compared to other Fluoroquinolones. Almost all the isolates were sensitive to Vancomycin \& Linezolid.

Similar findings were observed in Prakash et al study ${ }^{7}$ where Escherichia coli was the most common isolate among the uropathogens $(42.58 \%)$ followed by Klebsiella pneumoniae (18.71\%) Pseudomonas aeruginosa (12.90\%), Staphylococcus aureus $(9.68 \%)$. Carbapenems have shown very good activity for majority of the gram negative isolates in their study.

Staphylococcus aureus was the most common etiological agent isolated from wound infections (Pus \& Wound swabs) followed by E.coli, Klebsiella \& P seudomonas. No resistance was observed for Vancomycin \& Linezolid among Staphylococcus aureus. Resistance pattern of Gram negative isolates were almost similar to that of Urinary isolates. Similar findings were observed in different studies by various researches. ${ }^{8,9}$

Among Respiratory specimens (ET secretions, BAL, Sputum etc.,) Klebsiella was the most common organism followed by Pseudomonas, Acinetobacter, Streptococcus pneumoniae and others. Klebsiella and Pseudomonas are the common isolates of Sputum whereas almost all the isolates of Acinetobacter were from ET secretions. Klebsiella was highly resistant to amoxyclav and third generation cephalosporins. Carbapenems remained as the drugs of choice in majority of the gram negative respiratory isolates as the resistance was considerably lower to these group of drugs with the exception being acinetobacter where more than $50 \%$ of the isolates being resistant to carbapenems.

Among the blood cultures E.coli was the most common gram negative organism isolated followed by Klebsiella. Though coagulase negative staphylococci were the most common gram positive cocci isolated from the blood cutures, the significance of these organisms is questionable in majority of the cases as they are the most common 
contaminants encountered in the blood culture specimens. Pseudomonas and S.aureus were also isolated in significant number of cases. For the enterobacteriacea isolated from blood cultures, carbapenems were highly effective followed by cefaperazone sulbactum/piperacillin tazobactum and amikacin. $36 \%$ of the S.aureus isolates from the blood culture were MRSA. Similar findings were observed in many other studies from India. ${ }^{10,11}$ In Gohel et al study E.coli and Klebsiella were the most common gram negative isolates from blood culture and staphylococcus was the most common gram positive organism. Carbapenems were highly active against majority of the gram negative isolates where as for staphylococci vancomycin and linezolid were very effective. ${ }^{11}$

Overall Gram Negative isolates were highly sensitive to Polymyxins, Tigecycline, Carbapenems, Aminoglycosides, Piperacillin-Tazobactum, Cefaperazone-sulbactum, Fosfomycin, Nitrofurantoin (for Urine Only). Resistance to other Beta-lacta ms including Cephalosporins, amoxyclav and Fluoroquinolones, Co-trimoxazole was high. Gram Positive isolates were highly sensitive to Vancomycin \& Linezolid

\section{Conclusion}

Periodic Antimicrobial resistance surveillance is one of the important factors for improvising the standards of the health care. Understanding the local resistance patterns, systematic analysis and application of the surveillance data will help the clinicians in appropriate management of the patients \& also prevents the emergence of drug resistance among the clinical isolates.

\section{Source of Funding}

None.

\section{Conflict of Interest}

None.

\section{References}

1. Dixit A, Kumar N, Kumar S, Trigun V. Antimicrobial resistance: Progress in the decade since emergence of New Delhi metallo- lactamase in India. Indian J Community Med. 2019;44:4-8.

2. Walia K, Madhumathi J, Veeraraghavan B, Chakrabarti A, Kapil A, et al. Establishing Antimicrobial Resistance Surveillance \& Research Network in India: Journey so far. Indian J Med Res. 2019;149:164-79.

3. CLSI, "Performance standards for antimicrobial susceptibility testing," Twentieth informational supplement, Clinical and Laboratory Standards Institute Doc. M100ED28-2018. ; 2018,

4. Laxminarayan R, Duse A, Wattal C, Zaidi AK, Wertheim HF, Sumpradit N. Antibiotic resistance-the need for global solutions.; 2013,

5. Levy SB, Marshall B. Antibacterial resistance worldwide: causes, challenges and responses. Nature Med. 2004;10(12):122-129.

6. Sardar A. Comparative Evaluation of Fosfomycin Activity with other Antimicrobial Agents Against E.coli Isolate. . J Clin Diagn Res. 2017;11(2):26-29.

7. Prakash D, Saxena RS. Distribution and antimicrobial susceptibility pattern of bacterial pathogens causing urinary tract infection in Urban Community of Meerut City India. ISRN Microbiol. 2013;2013(13).

8. Mantravadi HB, Chinthaparthi MR, Shravani V. Aerobic isolates in pus and their antibiotic sensitivity pattern: a study conducted in a teaching hospital in Andhra Pradesh. Int J Med Sci Public Health. 2015;4:1076-1079.

9. Jamatia. Bacteriological Profile and Antimicrobial Resistance Patterns isolates in pus samples at Agartala Government Medical College. Asian J Pharm Clin Res. 2017;10(1):1-3.

10. Gupta S, Kashyap B. Bacteriological profile and antibiogram of blood culture isolates from a tertiary care hospital of North India. Trop $J$ Med Res. 2016;19:94-103.

11. Gohel K, Jojera A, Soni S, Gang S, Sabnis R, et al. Bacteriological profile and drug resistance patterns of blood culture isolates in a tertiary care nephrourology teaching institute. Biomed Res Int. 2014;p. 153747-153747.

\section{Author biography}

Chakrapani Kammineni Consultant Microbiologist

Naresh Yajamanam Consultant Biochemist and Assistant Professor

Amarnath K Reddy Consultant Biochemist

A. Mahammed Shafi Lab Technicians

Sreekanth Reddy Basireddy Assistant Professor

Cite this article: Kammineni C, Yajamanam N, Reddy AK, Shafi AM, Basireddy SR. Antimicrobial resistance surveillance of various clinical isolates in a diagnostic laboratory- An observational study. Indian J Microbiol Res 2019;6(3):202-207. 